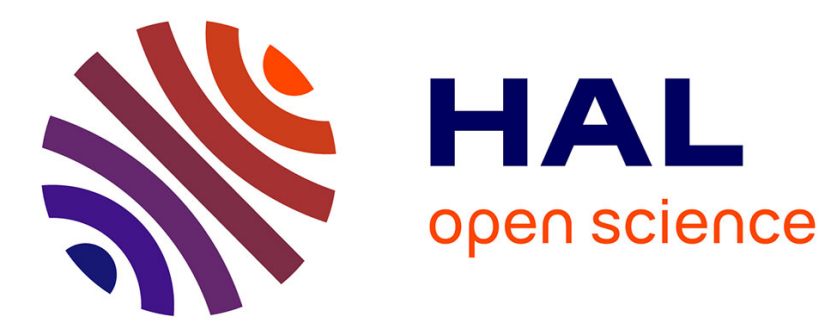

\title{
Pull-in and wrinkling instabilities of electroactive dielectric actuators
}

D de Tommasi, G Puglisi, G Saccomandi, G Zurlo

\section{To cite this version:}

D de Tommasi, G Puglisi, G Saccomandi, G Zurlo. Pull-in and wrinkling instabilities of electroactive dielectric actuators. Journal of Physics D: Applied Physics, 2010, 43 (32), pp.325501. 10.1088/0022$3727 / 43 / 32 / 325501$. hal-00569671

\section{HAL Id: hal-00569671 \\ https://hal.science/hal-00569671}

Submitted on 25 Feb 2011

HAL is a multi-disciplinary open access archive for the deposit and dissemination of scientific research documents, whether they are published or not. The documents may come from teaching and research institutions in France or abroad, or from public or private research centers.
L'archive ouverte pluridisciplinaire HAL, est destinée au dépôt et à la diffusion de documents scientifiques de niveau recherche, publiés ou non, émanant des établissements d'enseignement et de recherche français ou étrangers, des laboratoires publics ou privés. 


\title{
Pull-in and wrinkling instabilities of electroactive dielectric actuators
}

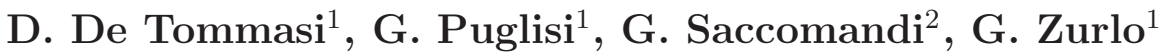 \\ ${ }^{1}$ Dipartimento di Ingegneria Civile e Ambientale, Politecnico di Bari, Italy \\ ${ }^{2}$ Dipartimento di Ingegneria Industriale, Università degli Studi di Perugia, 0612 \\ Perugia, Italy
}

\begin{abstract}
We propose a model to analyze the insurgence of pull-in and wrinkling failures in electroactive thin films. We take in consideration both cases of voltage and charge control, the role of prestretch and the size of activated regions, which are all crucial factors in technological applications of EAPs. Based on simple geometrical and material assumptions we deduce an explicit analytical description of these phenomena, allowing a clear physical interpretation of different failure mechanisms such as the occurrence of pull-in and wrinkling. Despite our simple assumptions, the comparison with experiments shows a good qualitative and, interestingly, quantitative agreement. In particular our model shows, in accordance with experiments, the existence of different optimal prestretch values, depending on the choice of the actuating parameter of the EAP.
\end{abstract}




\section{Introduction}

Electro-active polymers (EAPs) are very promising materials for several technological applications in the robotic, medical, energy harvesters, and biological fields [13, 12, 4]. These materials are characterized by important qualities such as lightweight, small size, low-cost, flexibility, fast response. The rapid technological advances in material science have recently improved EAPs performances in terms of their traditional limits, such as small actuation forces, low robustness and the requirement of very high electric fields. A prototypical device for electro-actuation based on EAPs is constituted by a thin layer of polymeric material sandwiched between two compliant electrodes. Application of a voltage to the two parallel electrodes generates an electric force (measured through the so called Maxwell stress [2]) which induces a compression of the layer; since the polymer is typically nearly incompressible, the resulting transversal extension is used as a mean of actuation.

A typical drawback in technological applications based on the described electroactivated films is the insurgence of different types of instability, leading to failure (e.g. [14] and [18]). One of the well known phenomena occurring in these devices is the so called pull-in instability. In order to describe this type of instability, one may consider a toy model composed by two rigid conducting plates connected by an insulated linear spring, with a rest distance equal to $d$. If the plates are subjected to a voltage $V$, the Coulomb forces between the plates will tend to attract them so that the spring will result compressed. It is easy to check that there exists a voltage threshold $V^{*}$ such that for larger voltages no more equilibrium is possible between the elastic and electric forces and the top plate slams onto the bottom plate. The resulting thumb rule used in EAPs and similar MEMS devices is that the critical distance associated with $V^{*}$ equals $d / 3$.

Clearly, the quantitative determination of the pull-in voltage is not a simple task for real deformable devices: the failure mechanism of electro-activated thin films depends not only on electro-elastic interactions, but also on the actuator shape and on purely electrical effects. A further purely mechanical failure mechanism in EAPs is represented by the insurgence of compressive stresses inside the polymer film. In typical actuation systems (e.g.[13],[18]) the polymeric film is constrained inside a rigid frame which serves to assembly the actuator, to provide electrical insulation, to transmit the actuation force, and to provide a pre-stretching of the polymer. Under the action of electric forces the constrained polymer film may undergo in-plane compressions, typically shed in evidence by the insurgence of a buckling type instability known as wrinkling in membrane theory $([19,22])$. Another crucial aspect for technological applications is given by the observation that the polymer film is tipically activated only in an internal region, due again to both assembly and insulation motivations.

In the recent past several theoretical and numerical analysis have been proposed in order to elucidate the causes of the various failure mechanisms of EAPs actuation devices. The importance of prestraining has been clearly evidenced in [13] and [17], where experimental observations have shown the existence of optimal prestraining 
values. An interesting analysis of the benefits of prestraining has been carried on in [11] for an unconstrained layer activated on the whole surface; in the same work, the necessity of a correct description of the strongly non linear material behavior of the polymer has also been evidenced. A similar problem is considered in [16] where the authors have discussed the relevance of an energetic analysis in the description of the pull-in instability. A numerical analysis for a circular planar actuator has been recently presented in [18], where the main role of prestretch and stretch rate up to failure are numerically analyzed. An energetic analysis for a homogeneously deformed dielectric elastomers with prestress and pull-in is studied in [23] and [3]. Finally, a theoretical analysis of the insurgence of deformation localization in a variational framework was recently proposed in [20] and [24], where the role of damage and dissipation were considered.

In this paper we propose a simple prototypical model for a slender prestrained actuator with an inner region activated by assigned voltage or charge (see the scheme in Fig.1). Based on simplifying geometrical and material hypotheses we elucidate the combined roles of prestrain, material properties and dimensions with respect to the wrinkling and pull-in failure modes. Due to the film thinness we neglect bending resistance and the formation of a boundary layer between activated and non activated regions, so that we assume a sharp interface between such regions $[6,5]$ and we ignore fringing effects [15]. Our geometrical assumptions are well suited for linear actuators typically adopted in artificial muscle technologies [17]. Of course our approach can be extended to more complex geometrical schemes and constitutive assumptions by using standards numerical methods. Moreover we do not consider dielectric strength thus neglecting the possibility of dielectric breakage [12].

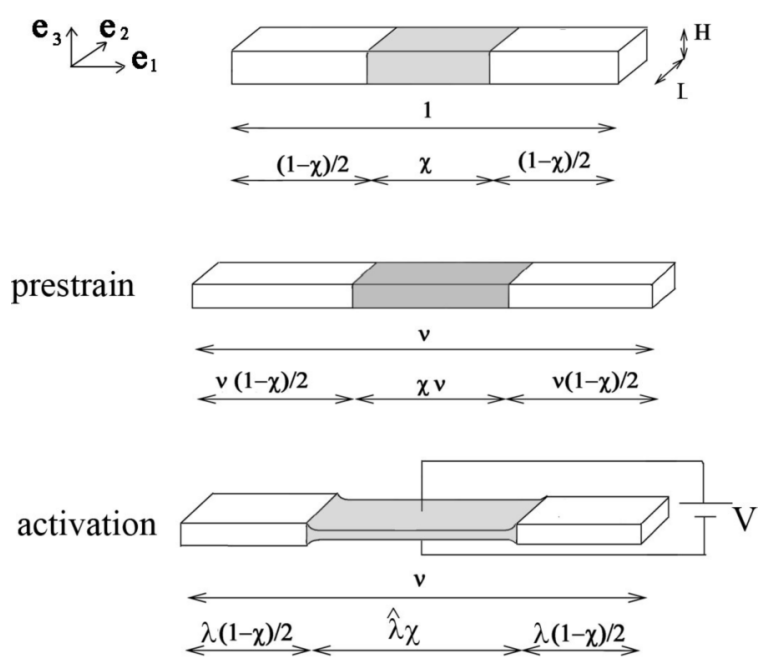

Figure 1. Scheme of the model and boundary conditions. The gray region represents the activated region.

The paper is organized as follows. In section 2 we recall the basic electromechanical 
equilibrium equations. In section 3 we introduce our prototypical example for a thin electroactivated system. In section 4 we obtain analytical solutions for both boundary value problems with assigned charge and voltage, as a function of given prestretch. Then, in section 5, we discuss the results of our model. In particular, we deduce analytically the existence of an optimal pre-strain (experimentally detected in [17]) which depends on the material modulus of the polymeric layer and on the size of the activated region. Shortly, the model describes how prestretch has a positive effect on the wrinkling instability and a negative effect on the pull-in instability, leading to the existence of an optimal prestretch. The corresponding value sof the activation parameters are then deduced. In section 6 we show that the model well describes the experimental behavior. Finally, in the Appendix we deduce a necessary stability condition for the electroactive device here considered.

\section{Basic Equations}

In this section we collect the main equations that are at the base of our theoretical approach. We refer the reader to the recent paper [2] and to the references therein for details. Let $\boldsymbol{X} \in \mathcal{B}_{0}$ and $\boldsymbol{x} \in \mathcal{B}$ be the typical point in the reference $\mathcal{B}_{0}$ and in the current configuration $\mathcal{B}$ of a continuous body, with $\boldsymbol{x}=\boldsymbol{f}(\boldsymbol{X})$, where $\boldsymbol{f}$ is the deformation field. Moreover, we denote by $\phi, \boldsymbol{D}$ and $\boldsymbol{E}$ the potential, the displacement vector and the electric field in the current configuration, respectively. For a linear, homogeneous and isotropic dielectric we have

$$
\boldsymbol{D}=\varepsilon \boldsymbol{E}=-\varepsilon \operatorname{grad} \phi,
$$

where $\varepsilon=\varepsilon_{0} \varepsilon_{d}$ with $\varepsilon_{0}$ the permittivity of free space and $\varepsilon_{d}$ the dielectric constant of the material. The equilibrium equations for the electromechanical problem are

$$
\begin{aligned}
& \operatorname{div} \boldsymbol{D}=\rho, \operatorname{div} \mathbf{T}=\boldsymbol{b} \text { in } \mathcal{B} \\
& \llbracket \boldsymbol{D} \rrbracket \cdot \boldsymbol{n}=\hat{q} \text { in } \partial \mathcal{B}_{q}, \mathbf{T} \boldsymbol{n}=\hat{\boldsymbol{s}} \text { in } \partial \mathcal{B}_{s} .
\end{aligned}
$$

Here $\rho$ and $\hat{q}$ (assigned on $\partial \mathcal{B}_{q}$ ) are the bulk and surface charge densities and $\boldsymbol{b}$ and $\hat{\boldsymbol{s}}$ (assigned on $\partial \mathcal{B}_{s}$ ) are the bulk and surface forces densities in the current configuration. $\mathbf{T}$ is the (symmetric) total stress tensor, that can be decomposed as the sum of the elastic (ela) term $\mathbf{T}^{e l a}$ and of the electric (elc) component $\mathbf{T}^{e l c}$ (i.e. the Maxwell stress)

$$
\mathbf{T}=\mathbf{T}^{e l a}+\mathbf{T}^{e l c},
$$

with

$$
\begin{aligned}
& \mathbf{T}^{e l c}=\varepsilon\left(\boldsymbol{E} \otimes \boldsymbol{E}-\frac{1}{2}(\boldsymbol{E} \cdot \boldsymbol{E}) \mathbf{I}\right), \\
& T_{i j}^{e l c}=\varepsilon\left(\frac{1}{2} E_{i} E_{j}-(\boldsymbol{E} \cdot \boldsymbol{E}) \delta_{i j}\right),
\end{aligned}
$$

where $i, j$ range from 1 to 3 (here and in the following we will use, when necessary, both component and absolute notations). The corresponding nominal (Piola-Kirchhoff) stress can be obtained as

$$
\mathbf{S}=\mathbf{S}^{e l a}+\mathbf{S}^{e l c}=J \mathbf{T}^{e l a} \mathbf{F}^{-T}+J \mathbf{T}^{e l c} \mathbf{F}^{-T},
$$


where $\mathbf{F}:=\nabla \boldsymbol{f}$ and $J:=\operatorname{det} \mathbf{F}$.

\section{Setting of the problem}

We consider a dielectric elastomer actuator which occupies, in its natural configuration, a right prismatic region $[0, \ell] \times[0, \tilde{L}] \times[0, \tilde{H}]$. We use the length $\ell$ of the elastomer to adimensionalize the thickness $H=\tilde{H} / \ell$ and the width $L=\tilde{L} / \ell$ and thus we consider the prismatic region $[0,1] \times[0, L] \times[0, H]$ (see Fig.1, where we also indicate the chosen coordinate system). In particular, we assume that the dielectric is thin, in the sense that $H \ll L \ll 1$. For thin films the membrane approximation of the nonlinear elasticity may be adopted. The standard membrane assumption (e.g. [10]) states that the bending stiffness is zero and any in-plane compressive stress leads immediately to the membrane buckling, with the appearance of a wrinkled regions. The presence of wrinkles is frequently encountered in experiments on thin dielectric elastomers and it represents one of the main observed failure mechanism of such devices.

We assume that voltage or charge can be controlled on a region of the thin film, more precisely on opposite strips of length $\chi \leq 1$ belonging to the upper and lower faces of the actuator. This region is placed centrally in direction of $\boldsymbol{e}_{1}$ length and it covers the full length $L$ in direction $\boldsymbol{e}_{2}$. The prismatic region of the actuator with upper and lower faces coinciding with the electrically controlled strips will be shortly denoted by active region, whereas the remaining part is simply called non active region. In accordance with the technological applications described in the introduction, we consider the possibility of a prestretch of the actuator along the $\boldsymbol{e}_{1}$ direction (see again Fig.1).

We describe the layer deformation by two homogeneous deformations with eigenvectors coinciding with the coordinate axes. More specifically, we assume in the non active region a deformation $f$ such that

$\underline{\text { non active region: }}$

$$
\begin{aligned}
& x_{1}=\lambda_{1} X_{1} \\
& x_{2}=\lambda_{2} X_{2} \\
& x_{3}=\lambda_{3} X_{3}
\end{aligned}, \quad \mathbf{F}:=\nabla \boldsymbol{f}=\left[\begin{array}{ccc}
\lambda_{1} & 0 & 0 \\
0 & \lambda_{2} & 0 \\
0 & 0 & \lambda_{3}
\end{array}\right]
$$

In the activated region we denote by $\hat{\boldsymbol{X}}=\left(\hat{X}_{1}, \hat{X}_{2}, \hat{X}_{3}\right)$ and $\hat{\boldsymbol{x}}=\left(\hat{x}_{1}, \hat{x}_{2}, \hat{x}_{3}\right)$ the generic point in the reference and current configuration, respectively, and by $\hat{f}$ the deformation. For this region we assume

$$
\begin{aligned}
& \text { active region: } \\
& \hat{x}_{1}=\hat{\lambda}_{1} \hat{X}_{1} \\
& \hat{x}_{2}=\hat{\lambda}_{2} \hat{X}_{2} \\
& \hat{x}_{3}=\hat{\lambda}_{3} \hat{X}_{3}
\end{aligned}, \quad \hat{\mathbf{F}}:=\nabla \hat{\boldsymbol{f}}=\left[\begin{array}{ccc}
\hat{\lambda}_{1} & 0 & 0 \\
0 & \hat{\lambda}_{2} & 0 \\
0 & 0 & \hat{\lambda}_{3}
\end{array}\right]
$$

The assumption of piecewise homogeneous deformation can be justified by the following hypotheses: piecewise homogeneous loading, thinness hypothesis, and 
constitutive assumption of homogeneous, isotropic, material behavior of the polymer. This type of deformations (which essentially neglects the presence of a geometricallycompatibile boundary layer at the interface between active and non active regions) is classical within the framework of phase-transitions [9] and of thin bodies subjected to homogeneous boundary loading (see, e.g., $[6,5]$ ). The balance equation at the interface may be formulated in the weak form $\llbracket \mathrm{S} \rrbracket \boldsymbol{e}_{1}=\boldsymbol{O}, \boldsymbol{e}_{1}$ being the normal to the interface in the reference configuration.

As well as the geometrical boundary layer we here neglect edge effects of the electric field, known in literature as fringing fields. Across the interface between the active and non active regions any discontinuity of the electric field $\llbracket \boldsymbol{E} \rrbracket$ must fulfill the jump condition (see e.g. [2]) $\boldsymbol{e}_{1} \times \llbracket \boldsymbol{E} \rrbracket=\boldsymbol{O}$. The assumption of negligible fringing fields leads to an apparent violation of the jump condition, since it results that $\boldsymbol{E}=E \boldsymbol{e}_{3}$ in the active region whereas $\boldsymbol{E}=\boldsymbol{O}$ in the non active region, so that the component of $\boldsymbol{E}$ which is parallel to the interface is not continuous through the interface; this apparent violation is actually resolved by considering that in reality the electric field undergoes an abrupt, but continuous, variation from $E$ to 0 in a region of negligible width. Also the assumption regarding electrical edge effects are acceptable, granted the thickness of the EAP is much smaller than its other dimensions (see e.g. [15]).

Regarding the polymer material behavior we assume that this is incompressible, so that its deformations respect the isochoric constraint

$$
\hat{\lambda}_{1} \hat{\lambda}_{2} \hat{\lambda}_{3}=1, \quad \lambda_{1} \lambda_{2} \lambda_{3}=1
$$

Moreover, in a typical application scheme, the actuator is pre-stretched in the direction $X_{1}$, say of an amount equal to $\nu$. By imposing that the total length of the actuator in the current configuration equals $\nu$, we get

$$
\nu=\chi \hat{\lambda}_{1}+(1-\chi) \lambda_{1}
$$

To obtain analytic results we assume that the incompressible polymer is characterized by a neo-Hookean constitutive response

$$
\begin{aligned}
& \mathbf{T}^{e l a}=-\tilde{\pi} \mathbf{I}+\mu \mathbf{B}, \quad \mathbf{S}^{e l a}=\mathbf{T}^{e l a} \mathbf{F}^{-T}, \\
& T_{i j}^{e l a}=-\tilde{\pi} \delta_{i j}+\mu \lambda_{i}^{2}, \quad S_{i j}^{e l a}=-\tilde{\pi} \lambda_{i}^{-1} \delta_{i j}+\mu \lambda_{i},
\end{aligned}
$$

where $\tilde{\pi}$ is a Lagrange multiplier due to the incompressibility constraint, $\mu$ is the shear modulus and $\mathbf{B}:=\mathbf{F F}^{T}$ is the left Cauchy-Green deformation tensor. By introducing the adimensionalized principal stresses and pressure

$$
s_{i}:=\frac{S_{i}}{\mu}, \quad t_{i}:=\frac{T_{i}}{\mu}, \quad \pi:=\frac{\tilde{\pi}}{\mu},
$$

the non zero stress components in the non active region can be calculated by substitution of the deformation (3.1) in the constitutive response, which gives

non active region:

$$
s_{i}=-\pi \lambda_{i}^{-1}+\lambda_{i}, \quad t_{i}=-\pi+\lambda_{i}^{2} \quad(i=1,2,3) .
$$


As anticipated above, we assume that the electric field in the active region coincides with $\boldsymbol{E}=(0,0, E)$. By using (2.3) and (2.4) the principal stresses in the active region are given by

$$
\begin{aligned}
& \frac{\text { active region: }}{\hat{t}_{i}=-\pi+\hat{\lambda}_{i}^{2}}+\varepsilon \frac{E^{2}}{\mu}\left(\delta_{i 3}-\frac{1}{2}\right), \\
& \hat{s}_{i}=-\pi \hat{\lambda}_{i}^{-1}+\hat{\lambda}_{i}+\varepsilon \frac{E^{2}}{\mu}\left(\delta_{i 3}-\frac{1}{2}\right) \hat{\lambda}_{i}^{-1} .
\end{aligned}
$$

The nature of the electric stresses, as well as their geometrical coupling with the deformation, changes according to the fact that voltage or electric charge are externally controlled by the device which drives the actuator. In the first case, the applied voltage $V$ determines the electric field:

$$
\text { voltage control: } \boldsymbol{D}=\left(0,0, \frac{\varepsilon V}{H \hat{\lambda}_{3}}\right), \boldsymbol{E}=\left(0,0, \frac{V}{H \hat{\lambda}_{3}}\right) \text {. }
$$

In the second case, the total electric charge $Q$ is controlled on the electrodes, so that

$$
\underline{\text { charge control }}: \boldsymbol{D}=\left(0,0, \frac{Q}{\hat{\lambda}_{1} \hat{\lambda}_{2} A}\right), \boldsymbol{E}=\left(0,0, \frac{Q}{\varepsilon \hat{\lambda}_{1} \hat{\lambda}_{2} A}\right) .
$$

Here $A=\chi L$ is the reference area where the charge is applied. In the following the two driving mechanisms will be considered separately.

\section{Equilibrium for the voltage and charge controls}

We first consider the following pre-stretch deformation $\boldsymbol{f}^{p}$ in the $\boldsymbol{e}_{1}$ direction

$$
\begin{aligned}
& x_{1}^{p}=\nu X_{1} \\
& x_{2}^{p}=\nu_{2} X_{2} \\
& x_{3}^{p}=\nu_{3} X_{3}
\end{aligned}, \quad \boldsymbol{F}^{p}:=\nabla \boldsymbol{f}^{p}=\left[\begin{array}{ccc}
\nu & 0 & 0 \\
0 & \nu_{2} & 0 \\
0 & 0 & \nu_{3}
\end{array}\right],
$$

where $\nu=\left(\nu_{2} \nu_{3}\right)^{-1}$ is the assigned longitudinal prestretch. (Here and in the following, for simplicity of notation, we sometime drop the index 1 when there is no ambiguity.)

The principal Cauchy stresses in the prestretched configuration are given by

$$
t_{i}^{p}=-\pi^{p}+\nu_{i}^{2}
$$

so that after imposing the conditions of vanishing stresses on the faces orthogonal to the axis $X_{2}$ and $X_{3}$

$$
t_{2}^{p}=t_{3}^{p}=0,
$$

we obtain $\pi^{p}=\nu^{-1}$ and $\nu_{2}=\nu_{3}=\nu^{-1 / 2}$. Thus, the only non-zero stress component in the prestretched configuration amounts to

$$
t_{1}^{p}=\nu^{2}-\nu^{-1}, \quad s_{1}^{p}=\nu-\nu^{-2} .
$$


Now we consider the electric activation of the EAP. Regarding the nonactive region the boundary conditions $t_{2}=t_{3}=0$ give $\left(\lambda_{1}, \lambda_{2}, \lambda_{3}\right)=\left(\lambda, \lambda^{-1 / 2}, \lambda^{-1 / 2}\right)$ so that the stress in this region as in Eq.ns (4.3) is given by

$$
t_{i}=\left(\lambda^{2}-\lambda^{-1}\right) \delta_{i 1}, \quad s_{i}=\left(\lambda-\lambda^{-2}\right) \delta_{i 1} .
$$

Concerning the electrically active region, after substitution of Eq.ns (3.8) in Eq.(3.7), we get

$$
\text { voltage control: } \hat{t}_{i}=-\hat{\pi}+\hat{\lambda}_{i}^{2}+\frac{1}{2 \mu} \frac{\varepsilon V^{2}}{\hat{\lambda}_{3}^{2} H^{2}}\left(2 \delta_{i 3}-1\right),
$$

and, by using (3.3), (3.7) and (3.9) we get

$$
\text { charge control: } \hat{t}_{i}=-\hat{\pi}+\hat{\lambda}_{i}^{2}+\frac{1}{2 \mu} \frac{Q^{2} \hat{\lambda}_{3}^{2}}{\varepsilon A^{2}}\left(2 \delta_{i 3}-1\right) \text {. }
$$

In order that the EAP maintains its total length equal to the given prestretch also after the application of charge or voltage (see Eq.(3.4)), we impose

$$
\nu=(1-\chi) \lambda+\chi \hat{\lambda} .
$$

This equation gives the following relation

$$
\lambda=\frac{\nu-\chi \hat{\lambda}}{1-\chi}
$$

so that the condition $\lambda>0$ implies that

$$
\hat{\lambda}<\nu / \chi
$$

Using (4.4) and (4.8) we then obtain

$$
s=\left(\frac{\nu-\chi \hat{\lambda}}{1-\chi}\right)-\left(\frac{\nu-\chi \hat{\lambda}}{1-\chi}\right)^{-2} .
$$

Let us now take in consideration the case when voltage is applied. In this case it is convenient to introduce the parameter $\kappa_{V}$ defined by

$$
\kappa_{V}=\frac{1}{2 \mu} \frac{\varepsilon V^{2}}{H^{2}},
$$

which represents the (adimensionalized) electric free energy density under the assigned potential. By imposing the boundary conditions $\hat{t}_{2}=\hat{t}_{3}=0$, after simple calculations we obtain the equilibrium stress and strains

$$
\underline{\text { voltage control: }}\left\{\begin{array}{l}
\hat{s}=\hat{\lambda}-\frac{1}{\hat{\lambda}^{2} \sqrt{1-2 \kappa_{V} \hat{\lambda}^{2}}}, \\
\hat{\lambda}_{2}^{2}=\frac{1}{\hat{\lambda} \sqrt{1-2 \kappa_{V} \hat{\lambda}^{2}}}, \\
\hat{\lambda}_{3}^{2}=\frac{\sqrt{1-2 \kappa_{V} \hat{\lambda}^{2}}}{\hat{\lambda}} .
\end{array}\right.
$$


When the total charge is assigned, after imposing the boundary conditions $\hat{t}_{2}=\hat{t}_{3}=0$ and by introducing the (adimensionalized) electric energy density $\kappa_{Q}$

$$
\kappa_{Q}=\frac{1}{2 \mu} \frac{Q^{2}}{\varepsilon A^{2}},
$$

we obtain

$$
\underline{\text { charge control: }}\left\{\begin{array}{l}
\hat{s}=\hat{\lambda}-\frac{\sqrt{1+2 \kappa_{Q}}}{\hat{\lambda}^{2}}, \\
\lambda_{2}^{2}=\frac{\sqrt{1+2 \kappa_{Q}}}{\hat{\lambda}}, \\
\lambda_{3}^{2}=\frac{1}{\hat{\lambda} \sqrt{1+2 \kappa_{Q}}} .
\end{array}\right.
$$

Finally, in order to deduce the equilibrium configurations we must impose the mechanical balance at the interface between the active and nonactive regions, which reads

$$
s=\hat{s} .
$$

This equation let us determine $\hat{\lambda}$ and, hence, the equilibrium configurations of the system.

\section{Wrinkling and pull-in instabilities}

The two failure mechanisms of wrinkling and pull-in will be considered separately for the cases of voltage and charge control.

\subsection{Voltage Control}

When voltage is assigned (see Fig.2) the equilibrium configurations, according with (4.13), (4.10), (4.11) correspond (if these exist) to the zeros of the function

$$
g(\hat{\lambda}) \equiv \underbrace{\hat{\lambda}-\frac{1}{\hat{\lambda}^{2} \sqrt{1-2 \kappa_{V} \hat{\lambda}^{2}}}}_{\hat{s}}-\underbrace{\left[\frac{\left(\frac{\nu-\chi \hat{\lambda}}{1-\chi}\right)^{3}-1}{\left(\frac{\nu-\chi \hat{\lambda}}{1-\chi}\right)^{2}}\right]}_{s},
$$

measuring the stress discontinuity at the interface. In order to obtain physically reasonable deformations (see $(4.9)$ and $\left.(4.11)_{1}\right)$ we are interested to the solutions of $g(\hat{\lambda})=0$ in the range $\left(0, \hat{\lambda}^{*}\right)$ where $\hat{\lambda}^{*}$ is the $\min \left\{1 / \sqrt{2 \kappa_{V}}, \nu / \chi\right\}$. We observe that the stress $s$ in (4.10) is a ( $\nu$-dependent) monotonic decreasing function of $\hat{\lambda}$, whereas an analysis of (4.11) shows that $\hat{s}$ is a $\left(\kappa_{V}\right.$-dependent) concave function of $\hat{\lambda}$, defined for $\hat{\lambda} \in\left(0,1 / \sqrt{2 \kappa_{V}}\right)$, with $\hat{s} \rightarrow-\infty$ at the boundaries of the definition domain. An inspection of the stress plots reported in Fig. $2_{a}$ shows that for given $\nu$ and $\chi$ the condition $g(\hat{\lambda})=0$ may have two roots $\hat{\lambda}_{-} \leq \hat{\lambda}_{+}$(equal or distinct) or no roots in the range of interest. If two roots exist it is possible to show that only one of them is stable in a 
sense which is discussed in detail in the Appendix; by means of this condition, it easy to check that the stable solution corresponds to the root $\hat{\lambda}_{-}$.

For given $\chi, \nu$ we define $\kappa_{V}^{\text {comp }}$ the value of the loading parameter in correspondence of which the stress evaluated at $\hat{\lambda}_{-}$is such that $s=\hat{s}=0$ (see Fig. $2_{b}$ ): for values of $\kappa_{V}>\kappa_{V}^{c o m p}$ the equilibrium stress is negative and, due to the lack of bending resistance, the film exhibits wrinkling, determining the device failure. Always keeping $\nu, \chi$ fixed, a second eventuality corresponds to values of the load parameter for which the two solutions coalesce (see again Fig. $2_{b}$ ), so that for values of $\kappa_{V}$ larger than this critical value $\kappa_{V}^{\text {pull }}$ no more roots of $s=\hat{s}$ exist: this instance represents the pull-in instability. For $\kappa_{V}>\kappa_{V}^{\text {pull }}$ the elastic response of the film is no more capable of balancing the compressive electric forces and the facing electrodes smash onto each other. Clearly, in correspondence of $\kappa_{V}=\kappa_{V}^{\text {pull }}$ it results $g=0$ and $d g / d \hat{\lambda}=0$.

The situation is depicted in Fig.3, where both values of $\kappa_{V}^{\text {comp }}$ and of $\kappa_{V}^{\text {pull }}$ are plotted with respect to the prestretch $\nu$. Under the particularly simple assumptions of our model, the expression of $\kappa_{V}^{c o m p}$ can be deduced analytically by imposing $s_{1}=\hat{s}_{1}=0$, which gives

$$
\kappa_{V}^{c o m p}(\nu)=\frac{1}{2}\left(\frac{1}{\hat{\lambda}^{2}(\nu)}-\frac{1}{\hat{\lambda}^{8}(\nu)}\right) \text {, with } \hat{\lambda}(\nu)=\frac{\nu+\chi-1}{\chi} .
$$

The function $\kappa_{V}^{\text {comp }}(\nu)$ is plotted with a bold line in Fig.3; it attains a maximum in correspondence of a prestretch

$$
\nu_{o p t}^{\text {comp }}=1+\left(2^{1 / 3}-1\right) \chi .
$$

The maximum value of $\kappa_{V}$ which can be applied to the film without inducing compression then amounts to

$$
\kappa_{V, \max }^{\text {comp }}=\frac{1}{2}\left(2^{-2 / 3}-2^{-8 / 3}\right)
$$

attained when

$$
\hat{\lambda}=\hat{\lambda}_{\text {opt }}^{\text {comp }}=2^{1 / 3} .
$$

The analytical expression of $\kappa_{V}^{\text {pull }}$ is not easy to get, but the numerical solution of the conditions $g=0, d g / d \hat{\lambda}=0$ gives $\kappa_{V}^{\text {pull }}$ for each value of $\nu$, which is represented by a light line in Fig.3. It is possible to show that, for fixed $\chi$, the curve $\kappa_{V}^{\text {comp }}(\nu)$ is always under the curve $\kappa_{V}^{\text {pull }}(\nu)$ but in a common point where these curves are tangent, corresponding to the simultaneous attainment of both wrinkling and pull-in failures. This point can be analytically determined by imposing that $s=\hat{s}=0$ and $d g / d \hat{\lambda}=0$, which after simple algebraic manipulations yields the value of the prestretch

$$
\nu_{o p t}^{\text {pull }}=\chi\left(\hat{\lambda}_{o p t}^{\text {pull }}(\chi)-1\right)+1
$$

where

$$
\hat{\lambda}_{o p t}^{\text {pull }}(\chi):=\left(\frac{4-\chi}{1-\chi}\right)^{1 / 6}
$$




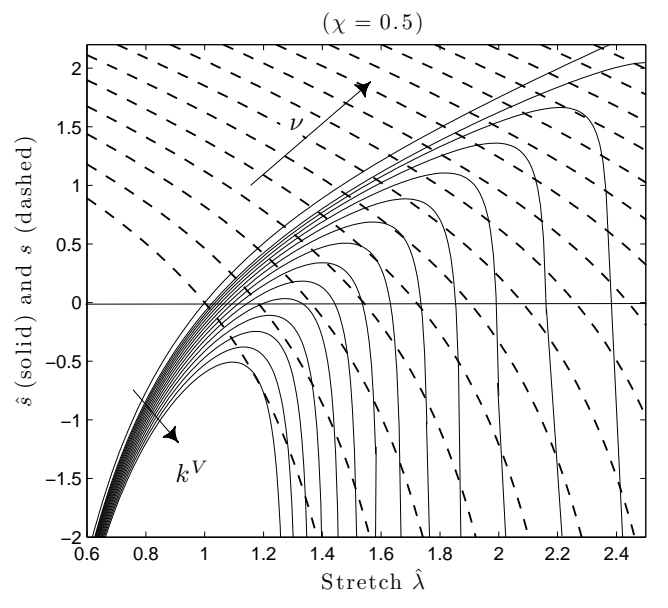

a)

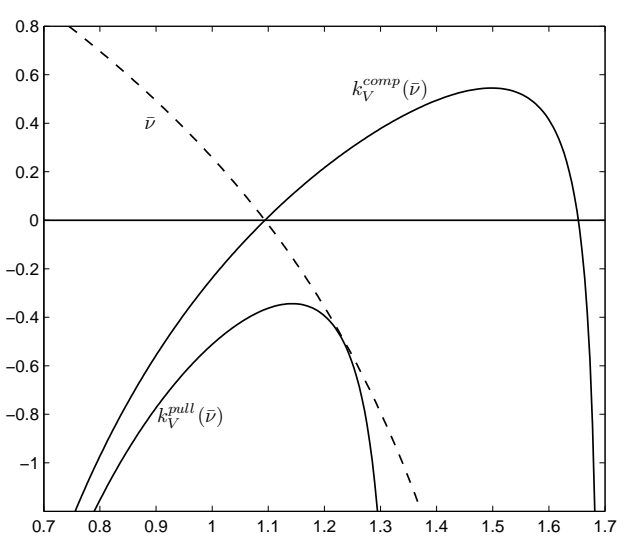

b)

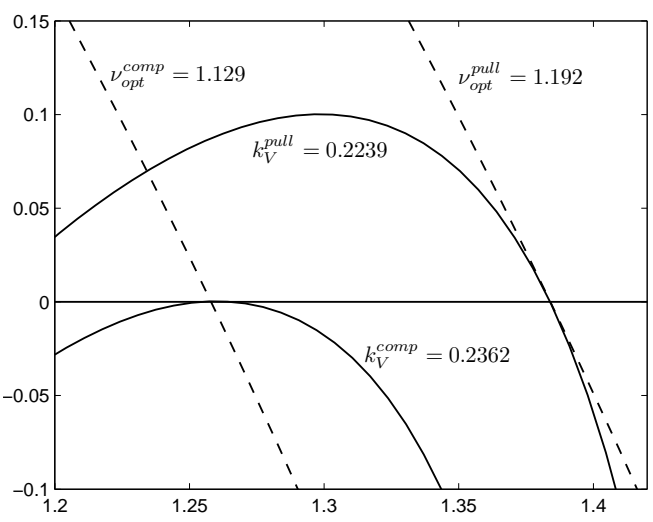

c)

Figure 2. Stress strain curves in the activated and non activated region in the case of assigned voltage: continuous lines represent the stress $\hat{s}_{1}\left(\hat{\lambda}, \kappa_{V}\right)$ in (4.11), of the activated region, whereas dashed lines represent the stress $s_{1}(\hat{\lambda}, \nu)$ in (4.10) in the non active region. In b) we show the attainment, for a fixed $\nu$ of both the pull-in and wrinkling instability. In c) we show the stress curves $s_{1}(\hat{\lambda}, \nu)$ corresponding to the maximum attained value of $\kappa_{V}$

It is easy to check that for all $\chi$ it results $\nu_{o p t}^{\text {comp }} \leq \nu_{o p t}^{\text {pull }}$.

It is interesting to observe that not all the values of the functions $\kappa_{V}^{\text {pull }}(\nu)$ and $\kappa_{V}^{\text {comp }}(\nu)$ are actually attainable; regarding $\kappa_{V}^{\text {pull }}(\nu)$, the analysis of the functions $s(\hat{\lambda})$ and $\hat{s}(\hat{\lambda})$ shows that for $\nu>\nu_{o p t}^{\text {pull }}$ the pull-in failure is attained at positive stress, whereas for $\nu<\nu_{o p t}^{\text {pull }}$ it is attained at negative stress, which is not possible in our model (dashed light line in Fig.3). On the other side it is possible to check that the values of $\kappa_{V}^{\text {comp }}$ corresponding to $\nu>\nu_{\text {opt }}^{\text {pull }}$ are relative to the unstable solutions $\hat{\lambda}_{+}$and hence these are not attainable (dashed bold line in Fig.3). As a result, the film failure is due to wrinkling for $\nu<\nu_{o p t}^{\text {pull }}$ and it is due to pull-in for $\nu>\nu_{o p t}^{\text {pull }}$. As it is clear from the plot, the highest value of the load parameter which can be applied without generating failure of both types coincides with the maximum value of $\kappa_{V}^{\text {comp }}$ given in Eq.5.2, which is attained in correspondence of $\nu=\nu_{o p t}^{\text {comp }}$; in this sense $\nu_{o p t}^{\text {comp }}$ is optimal if one is interested 


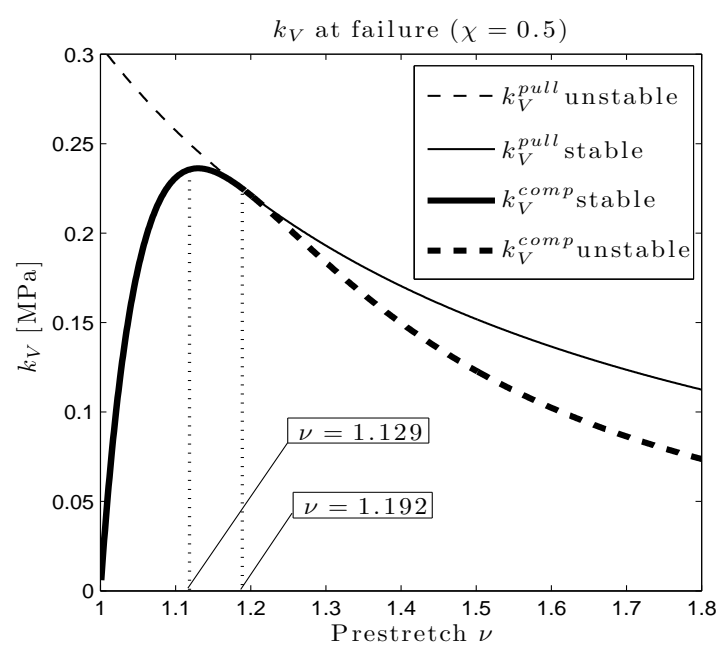

Figure 3. Critical values of the activation energy $k_{V}$. Bold line represents the function $k_{V}^{\text {comp }}(\nu)$ corresponding to wrinkling instability, whereas light line represent the function $k_{V}^{\text {pull }}(\nu)$ corresponding to pull-in instability. Dashed lines correspond to unstable configurations.

at the maximization of the stored electric energy.

A remarkable fact is that if one is interested at other useful activation parameters, such as the maximum actuating force or the maximum applicable electric field, then $\nu_{o p t}^{\text {comp }}$ is no more optimal. This can be seen by evaluating the values of the stress gap with respect to the prestretched configuration, given by $\Delta S:=\mu[\hat{s}(\nu)-\hat{s}(\hat{\lambda})]$ and of the electric field $\boldsymbol{E}$ in correspondence of the initiation of both wrinkling and pull-in failures; as it is evident from the plots, the maximum values of both parameters are now attained for $\nu=\nu_{o p t}^{\text {pull }}$, that is for $\hat{\lambda}=\hat{\lambda}_{\text {opt }}^{\text {pull }}$ (see $\operatorname{Eqn.s}(5.4)-(5.3)$ ). We remind that this value of $\nu$ corresponds to the simultaneous attainment of the wrinkling and pull-in failures. After a short calculation, we deduce that the optimal value of $\Delta S$

$$
\Delta S_{o p t}^{\text {pull }}=\mu\left(\nu_{o p t}^{\text {pull }}-\left(\nu_{o p t}^{\text {pull }}\right)^{-2}\right)
$$

whereas the optimal value of the electric field is given by

$$
E_{\text {opt }}^{\text {pull }}=\sqrt{\frac{3 \mu}{\varepsilon}}\left((1-\chi)^{-\frac{1}{6}}(4-\chi)^{-\frac{1}{3}}\right)
$$

It should be underlined, in conclusion, that all the activation parameters $\Delta S, \kappa_{V}$ and $\boldsymbol{E}$ depend on the elastic modulus $\mu$, and that $\Delta S$ and $\boldsymbol{E}$ grow with the fraction $\chi$ of activated film. The resulting dependence of $\nu^{\text {pull }}$ and $\nu^{\text {comp }}$ with respect to the fraction of activated material is summarized in Fig.5. 

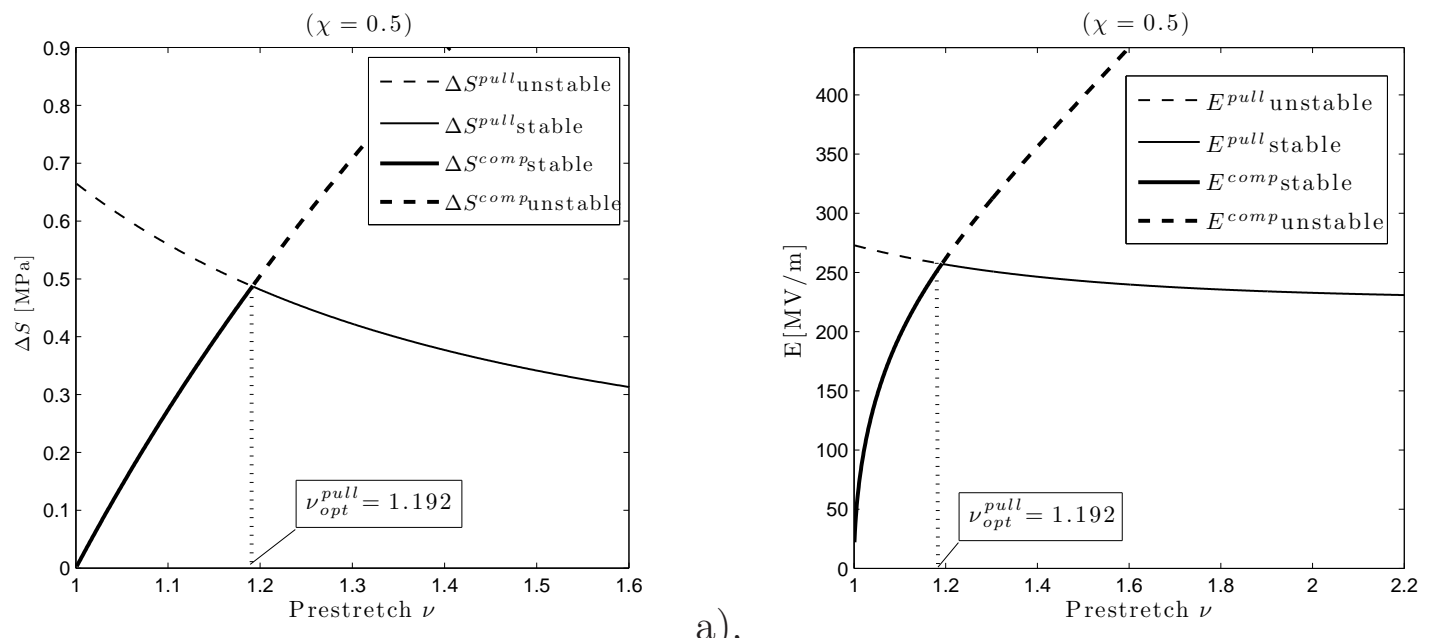

a),

Figure 4. Critical values of the activation stress $\Delta S$ a) and of the electric field $E$. Bold lines represent the values corresponding to wrinkling instability, whereas light lines represent the values corresponding to pull-in instability. Dashed lines correspond to unstable equilibrium states.

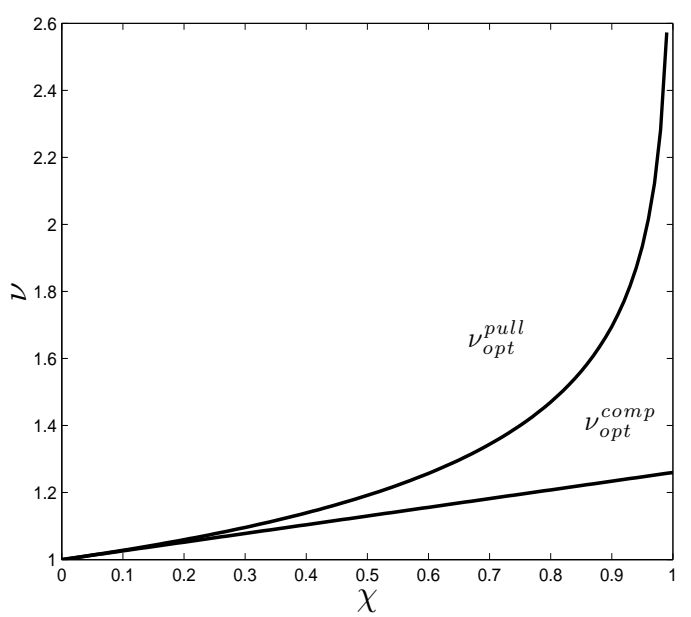

Figure 5. Optimal prestretch (5.1) and (5.3) as functions of the size $\chi$ of the activation region.

\subsection{Charge Control}

In the case of charge control, it is interesting to observe that the pull-in failure is not possible (this is in accordance with previous analyses [21]). This is due to the fact that for any $\nu$ and $\chi$ the stress $s$ is a decreasing function of $\hat{\lambda}$, whereas the stress $\hat{s}$ is an increasing function of $\hat{\lambda}$. On the contrary the wrinkling failure is possible and it is 
attained as soon as $s(\nu, \chi)=\hat{s}\left(\kappa_{Q}\right)=0$, that is

$$
\hat{\lambda}=\hat{\lambda}_{o p t}^{\text {comp }}(\nu)=1+\frac{\nu-1}{\chi}
$$

which corresponds to a maximum load parameter

$$
\kappa_{Q, \max }^{c o m p}=\frac{1}{2}\left(\left(\hat{\lambda}_{\text {opt }}^{\text {comp }}\right)^{6}-1\right) .
$$

Since the load parameter is an increasing function of $\nu$, it is clear that in the case of charge control it is not possible to find an optimal value of the prestretch.

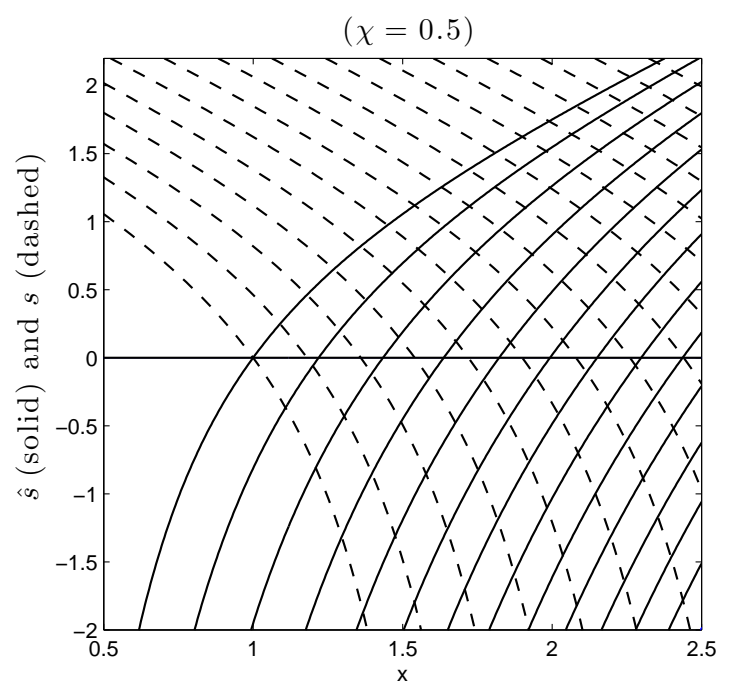

Figure 6. Stress strain curves in the activated and non activated region in the case of assigned charge: continuous lines represent the stress $\hat{s}_{1}\left(\hat{\lambda}, \kappa_{Q}\right)$ in $(4.12)$, of the activated region, whereas dashed lines represent the stress $s_{1}(\hat{\lambda}, \nu)$ in (4.10) in the non active region.

\section{Comparison with experiments}

In this section we briefly compare the theoretical results of our model with the experimental behavior of electroactive polymer actuators [13].

In Fig. $7_{a}$ we reproduce the experimental behavior of silicone rubber films (Nusil CF19-2186) as reported in [13], where circles represent the values of the electric field at failure for different assigned prestretches. In Fig. $7_{b}$ we reproduce the results of our model, where we have considered an elastic modulus $\mu=1 M P a$, as suggested by [13] for this material. It should be remarked that the considered experiments refer to circular actuators, whereas we here have focused attention on linear actuators. Nevertheless, the comparison between experiments and theoretical results are in nice quantitative agreement. 

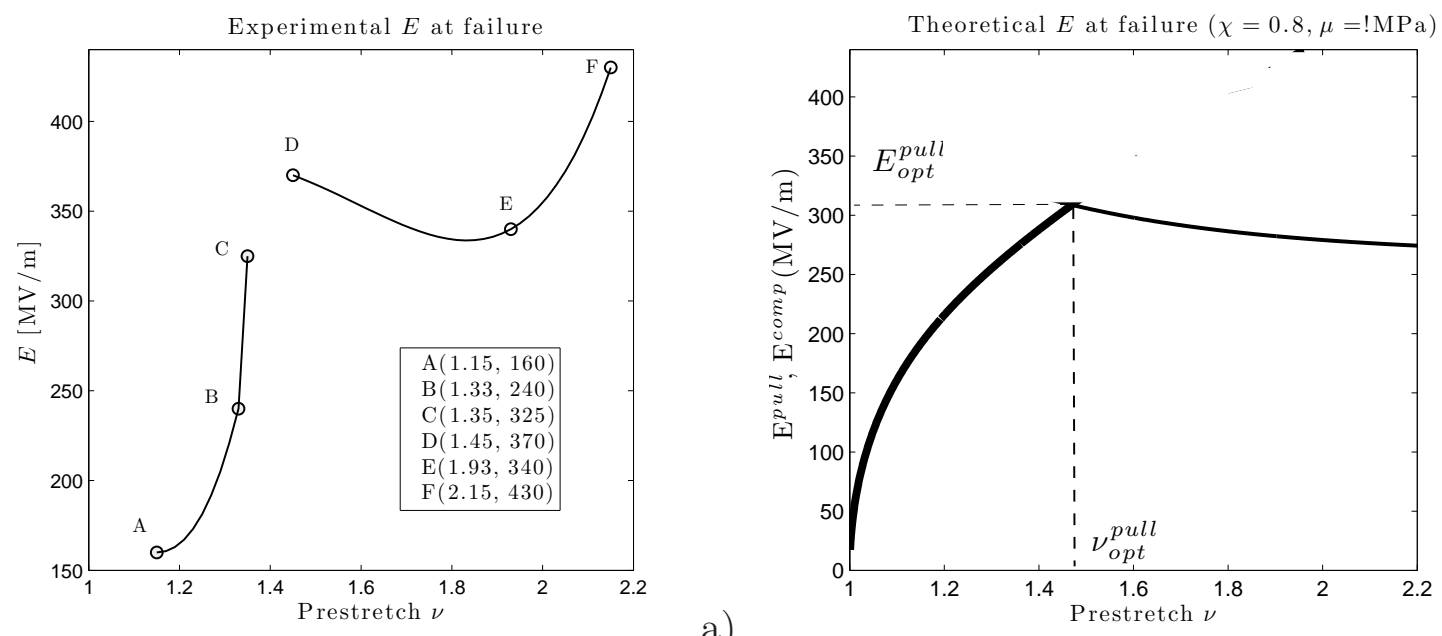

Figure 7. Failure value of the electric field as a function of the prestretch: a) experiment (deduced from [13]), b) theoretical.

Quite interestingly, a careful analysis of the experimental Fig. $7_{a}$ in light of the theoretical Fig. $7_{b}$ shows the existence of different behaviors, separated by a threshold prestretch which approximately corresponds to $\nu_{o p t}^{\text {pull }}$. For values of $\nu$ lower than $\approx 1.4$ both the experimental and the theoretical results show an initial growth of the maximum applicable electric field with growing prestretch. According to our model this may be due to the initially positive effect of prestretch in avoiding the occurrence of wrinkling. Such positive effect, both in experiments and in our model, ceases to exist when the prestretch overcomes the critical value of about 1.4, since here the thickness reduction anticipates the occurrence of pull-in failure. A finer description of the film behavior for larger values of the prestretch certainly requires a better description of the exact film geometry and of the material response. To the price of renouncing to closed form solutions (which are generally possible under very simple assumptions such those embraced in this article), the methods we have discussed can be numerically applied to more complex geometries and material behaviors suitable for polymers undergoing large deformations, such as Mooney-Rivlin, Gent, Arruda-Boyce and others; some of these applications will be considered in a forthcoming paper.

\section{APPENDIX}

In this Appendix a necessary stability condition is obtained for the equilibrium configurations of a linear actuator undergoing piecewise homogeneous deformations. We observe that the homogeneity of the deformations in both the active and non active regions, together with the constraint of assigned prestretch $\nu$, essentially reduces the present equilibrium problem to a one-dimensional problem. Under conservative hypotheses, the total energy $\mathscr{E}$ stored in the device at given $\hat{\lambda}$ can be obtained by integration of the internal working density along an arbitrary deformation path from 
the undeformed configuration; since in this case the only non zero stress component is $s$ we have

$$
\frac{\mathscr{E}(\hat{\lambda})}{H L}=\left[\chi \int_{1}^{\hat{\lambda}} \hat{s}(x) d x+(1-\chi) \int_{1}^{\lambda} s(x) d x\right] .
$$

This function must be minimized under the compatibility constraint $\nu=\chi \hat{\lambda}+(1-\chi) \lambda$, that gives

$$
\lambda=\lambda(\hat{\lambda})=\frac{\nu-\chi \hat{\lambda}}{1-\chi}
$$

Let then $\hat{\lambda}$ define a given configuration of the system, and let $\hat{\lambda}+\zeta$ be a perturbed configuration that is obtained by shifting the interface between the active and non active regions. The perturbed energy is

$$
\frac{\mathscr{E}(\hat{\lambda}+\zeta)}{H L}=\chi \int_{1}^{\hat{\lambda}+\zeta} \hat{s}(x) d x+(1-\chi) \int_{1}^{\lambda-\zeta \frac{\chi}{1-\chi}} s(x) d x .
$$

Thus, a stable configuration $\hat{\lambda}$ must verify

$$
\mathscr{E}(\hat{\lambda}+\zeta) \geq \mathscr{E}(\hat{\lambda}) \quad \text { for all } \zeta
$$

By considering the following expansion

$$
\begin{aligned}
\mathscr{E}(\hat{\lambda}+\zeta) & =\mathscr{E}(\hat{\lambda})+\zeta \chi[\hat{s}(\hat{\lambda})-s(\lambda)] \\
& +\chi \frac{\zeta^{2}}{2}\left[\frac{d \hat{s}(\hat{\lambda})}{d \hat{\lambda}}-\frac{d s(\lambda(\hat{\lambda}))}{d \hat{\lambda}}\right]+o\left(\zeta^{2}\right)
\end{aligned}
$$

the vanishing of the first variation gives the Euler Lagrange equilibrium condition

$$
\hat{s}(\hat{\lambda})=s(\lambda)
$$

whereas the positivity of the second variation gives

$$
\frac{d \hat{s}(\hat{\lambda})}{d \hat{\lambda}} \geq \frac{d s(\lambda(\hat{\lambda}))}{d \hat{\lambda}}
$$

which is the necessary stability condition adopted in the paper.

\section{References}

[1] J. Block, D.G. LeGrand. Dielectric breakdown of polymer films, J. Appl. Phys. 40, 288-293, 1969.

[2] R. Bustamante, A. Dorfman, R.W. Ogden. Nonlinear Electroelastostatics: a variational framework. Z. angew. Math. Phys., 60, 154-177, 2009.

[3] R. Díaz-Callja, M.J. Sanchis, and E. Riande. Effect of an electric field on the bifurcation of a biaxially stretched incompressible slab rubber. Eur. Phys. J. E, 39, 417-426, 2009.

[4] F. Carpi, D. De Rossi, R- Kornbluh, R. Perline, and P. Sommer-Larsen Ed. Dielectric elastomers as electromechanical transducers. Elsevier, 2008. 
[5] I. Carr, M.E. Gurtin, and M.Slemrod. Structured phase transition on a finite interval. Arch. Rat. Mech. Anal., 86, 317-351, 1984.

[6] B.D. Coleman. Necking and Drawing in Polymeric Fibers Under Tension, Arch. Rat. Mech. An. 83(2), 115-137, 1983.

[7] D. De Tommasi, G. Puglisi, G. Saccomandi. A micromechanics based model for the Mullins effect. J. Rheology, 50, 495-512, 2006.

[8] D. De Tommasi, G. Puglisi, G. Saccomandi. Localized versus Diffuse Damage in Amorphous Materials. Phys. Rev. Lett., 100, 085502, 2008.

[9] Y. B. Fu, A. B. Freidin. Characterization and stability of two-phase piecewise-homogeneous deformations. Proc. R. Soc. Lond. , 460, 3065-3094, 2004.

[10] D. M. Haughton and R. W. Ogden. On the incremental equations in non-linear elasticity-Membrane theory. J. Mech. Phys. Solids. 26, 93-110, 1978.

[11] G. Kofod. The static actuation of dielectric elastomer actuators: how does pre-stretch improve actuation. J. Phys. D: Appl. Phys., 41, 215405, 2008.

[12] S. Koh, X. Zhao, and Z. Suo. Maximal energy that can be converted by a dielectric elastomer generator. Appl. Phys. Lett., 94, 262902, 2009.

[13] R. Kornbluh, R. Perline, Q. Pei, S. Oh, J. Joseph. Ultrahigh strain response of field-actuated elastimeric polymers. Multibody System Dynamics, 1, 149-188, 1997.

[14] M. Moscardo, X. Zhao, Z. Suo, Y. Lapusta. On designing dielectric elastomers. J. Appl. Phys., 104, 093503, 2008.

[15] G. W. Parker. Electric field outside a parallel plate capacitor. Am. J. Phys., 70, 502-507, 2002.

[16] L. Patrick, K. Gabor, and M. Silvain. Characterization of dielectric elastomer actuators based on a hyperelastic film model. Sensors and Actuators A, 135, 748-757, 2007.

[17] R. Perline, R. Kornbluh, Q. Pei, and J. Joseph. High speed electrically actuated elastomers with strain greater that 100\%. Science, 287, 836-839, 2000.

[18] S. Plante, S. Dubowsky. Large-scale Failure modes of dielectric elastomer actuators. Int. J. Sol. Struct., 43, 7727-51, 2006.

[19] A.C. Pipkin. The relaxed energy density for isotropic elastic thin layers. IMA J. Appl. Math., 36, 85-99, 1986.

[20] G. Puglisi. Damage localization and stability in electroactive polymers. Report of Mathematisches Forschungsinstitut Oberwolfach, 10, 2008.

[21] J.I. Seeger and B.E. Boser. Charge control of parallel-plate, electrostatic actuators and the tip-in instability. J. Microelectromechanical Syst., 12, 2003.

[22] D.J. Steigmann. Tension-field theory. Proc. R. Soc. Lond. A, 429, 141-173, 1990.

[23] X. Zhao and Z. Suo. Method to analyze electromechanical stability of dielectric elastomers. J. Appl. Phys., 91, 061921, 2007.

[24] X. Zhao, W. Hong and Z. Suo. Electromechanical hysteresis and coexistent states in dielectric elastomers. Phys. Rev. B, 76, 134113, 2007. 